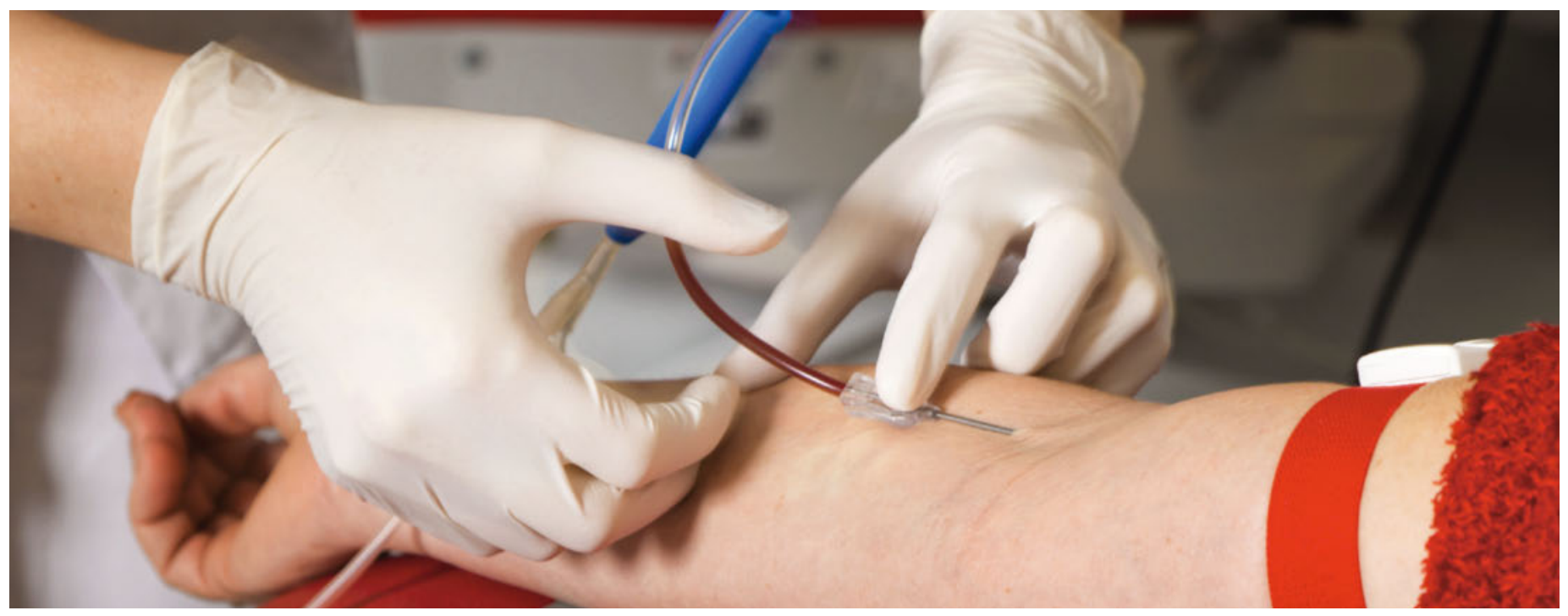

\title{
Aderlass - ein lukratives, unlauteres, aber risikoloses Geschäftsmodell
}

\section{Christian Marti}

Dr. med., Facharzt für Allgemeine Innere Medizin

Beschrieben wird der Einzelfall eines Kollegen, der eindeutig falsch und zu viel abrechnet. Dieser Fall erlaubt zwar keine Rückschlüsse darauf, wie gravierend oder wie marginal die geschilderte Problematik für das Gesamtsystem ist, wirft aber doch die Frage auf, wie wir unter Kollegen damit umgehen.

Als Mitglied eines Ärztenetzes, das eine gewisse Budgetmitverantwortung trägt, kann ich bei Bedarf praxisexterne Leistungsabrechnungen, die meine Patienten

\section{Aderlass ist eine banale, seit Jahrhunderten prak-} tizierte Massnahme - und risikolos bei einem kreislaufgesunden Mann mittleren Alters.

betreffen, einsehen. Dabei ist mir kürzlich die Abrechnung für eine Aderlass-Behandlung an einer Privatklinik aufgefallen. Darum habe ich den Patienten, der an einer Eisenspeicherkrankheit leidet, kontaktiert und von ihm erfahren, dass ...

- die gesamte Aderlassprozedur kaum je länger als eine halbe Stunde dauert,

- der ärztliche Kontakt zwischen dem behandelnden Hämatologieprofessor und dem Patient jeweils eher 5 als 10 Minuten dauert,

- die Aderlässe ausnahmslos durch medizinisches Hilfspersonal durchgeführt werden.
Den Abrechnungsbelegen dagegen ist zu entnehmen, dass systematisch ...

- ein Mehrfaches der Konsultationszeit des Arztes und deutlich längere Überwachungszeiten durch das Fachpersonal verrechnet werden, als sie den Angaben des Patienten entsprechen,

- bei jedem Aderlass, d.h. ein- bis zweimal pro Monat, mehrere Laborpositionen verrechnet werden, die sich ausnahmslos im Normbereich bewegen und keinerlei Bedeutung für die Behandlung haben,

- die Tarifposition "Aderlass durch den Facharzt» verrechnet wird, was nur dann erlaubt ist, wenn dieser durch den Facharzt persönlich durchgeführt wird.

Was mir wie Betrug und Mengenausweitung erscheint, rechtfertigt der Kollege schriftlich damit, dass er am Vortag des Aderlasses die Verordnung nochmals überprüfe. Am Tag der Behandlung finde «eine vorbereitende Sitzung mit dem Pflegepersonal» statt, wo «jeder Fall nochmals einzeln diskutiert und die Massnahmen 
im Team abgesprochen werden". Am Abend werden die Blutwerte dann von Pflege und Arzt kontrolliert und diskutiert.

Aderlass ist eine banale, seit Jahrhunderten praktizierte Massnahme - und risikolos bei einem kreislaufgesunden Mann mittleren Alters. Technisch entspricht der Ablauf heutzutage genau demjenigen beim Blutspenden. Der hier beschriebene bzw. behauptete und verrechnete Aufwand ist grotesk, ineffizient und führt insgesamt zu einer Verdoppelung der Kosten. (Ein beträchtlicher Teil davon müsste korrekterweise als «ärztliche Leistung in Abwesenheit des Patienten» und nicht als Konsultations- und Überwachungszeit abgerechnet werden.)

Die Tarifposition "Aderlass durch den Facharzt» darf nicht verrechnet werden, wenn dieser durch eine Hilfsperson durchgeführt wird, was auch der Tarifdienst der FMH schriftlich bestätigt. Im Widerspruch dazu behauptet der Kollege, sowohl «in Übereinstimmung mit internen und externen Kollegen als

\section{Die Tarifposition "Aderlass durch den Facharzt"} darf nicht verrechnet werden, wenn dieser durch eine Hilfsperson durchgeführt wird.

auch mit onkologischen Fachgruppen", dass diese Verrechnung korrekt sei, weil diese Therapie «vom Facharzt indiziert, überwacht und letztendlich verantwortet» werde. Das riecht entweder nach einer Schutzbehauptung oder nach illegaler Absprache.

Der schriftliche Rechtfertigungsversuch für diese Leistungsabrechnung zeigt Elemente eines ebenso lukrativen wie unlauteren, aber risikoarmen Geschäftsmodells:

1. Verrechnung nicht erbrachter Leistungen: Im Beispiel wird die pflegerische Handlung "Aderlass» als teurere ärztliche Leistung abgerechnet. Die verrechneten Konsultations- und Überwachungszeiten überschreiten den effektiven Zeiteinsatz bei weitem.

2. Mengenausweitung: Im Beispiel die engmaschigen, umfangreichen, aber bedeutungslosen Laborkontrollen.

3. Dramatisierung des Banalen: Im medizinischen Alltag ist sie allgegenwärtig. Viele Leistungserbringer fördern sie nach Kräften durch Übertreiben von Risiken und Chancen. Dies begünstigt nicht nur übertriebene Erwartungen, sondern auch Verunsicherung und Angst bei den Laien (die man postwendend als «begehrlich» beschimpfen kann). Ein schmerzhafter Insektenstich, ein kleine Marschoder Brandblase, eine leichte Erkältung oder eine flüchtige Befindlichkeitsstörung führt umgehend zum Arzt - oft als Notfall. Und aus einer simplen venösen Blutentnahme (Aderlass) und der Interpretation eines Eisenwertes entwickelt sich in Spezialistenhänden eine Aufgabe, die Spezialist und Patient einem Hausarzt kaum mehr zutrauen.

Dieses Geschäftsmodell ist nahezu risikolos, denn die Krankenversicherungen sind diesen Mechanismen aus folgenden Gründen weitgehend ohnmächtig ausgeliefert:

- Die Verrechnung nicht erbrachter Leistungen wird selten entdeckt. Am ehesten könnte dies noch der Patient, falls er überhaupt noch eine Rechnungskopie zu Gesicht bekommt und die Rechnung auch interpretieren könnte.

- Mengenausweitung eines einzelnen Arztes ist für die Krankenversicherung nur erkennbar, wenn er sie in grossem Stil betreibt. Ganz verborgen bleibt sie, wenn sich ganze Gruppen von Leistungserbringern auf eine selbstherrliche Tarifauslegung einigen.

- Die Dramatisierung des Banalen, oft unter dem Mäntelchen des medizinischen Fortschrittes, ist das grösste Problem, weil sie zunehmend Bestandteil des medizinischen Alltags wird. Die weitere Vertiefung dieses Themas würde den Rahmen dieses Beitrags sprengen.

Weil das Risiko, erwischt zu werden, minim ist, sollten Sanktionen umso schmerzhafter sein. Im Wiederholungsfall sollten Krankenversicherungen z.B. die Möglichkeit bekommen, Fehlbaren eine Karenzzeit aufzuerlegen, während welcher sie keine Leistungen zu Lasten der Sozialversicherungen abrechnen dürfen. Beschrieben wird hier der Einzelfall eines Kollegen, der eindeutig falsch und zuviel abrechnet. Aus seiner Sicht scheint es so, dass eine einfache Aderlass-Behandlung bei einem weitgehend gesunden Menschen ihm einen derart grossen intellektuellen und logistischen Aufwand abverlangt, dass die Behandlung am Ende doppelt so viel kostet wie bei einem Hausarzt. Dieser Einzelfall erlaubt natürlich keine Rückschlüsse darauf, wie gravierend odere wie marginal die geschilderte Problematik für das Gesamtsystem ist.

Wo wir Ärzte Einsicht in Leistungsabrechnungen haben, z.B. in Budgetmodellen, sollten wir bei Auffälligkeiten hin- statt wegschauen und den Kollegen ein Feedback geben. Wo die Abrechnungen trotzdem nicht nachvollziehbar bleiben, sollten wir sie aus dem Netz ausschliessen. Zuweisungen an netzexterne Kollegen, die das System trotz Feedback mit eindeutiger Mengenausweitung oder gar Verrechnung nicht erbrachter Leistungen zur Ader lassen, sollten ebenso obsolet sein wie Zuweisungen an fachlich zweifelhafte Kollegen.

Bildnachweis

(c) Ginasanders | Dreamstime.com 